\title{
Incorporación a la currícula de anestesia los bloqueos regionales ecoguiados en Uruguay
}

\author{
Ultrasound regional blocks in the anesthesia curricula \\ in Uruguay
}

Gustavo Ramos ${ }^{1}$, Juan Pablo Bouchacourt², Alejandro Corujo A. ${ }^{3}$, Luis Aren ${ }^{3}$

\section{E} n esta última década, la incorporación de la ecografía para la realización de procedimientos de anestesia regional ha permitido mejorar la comprensión, comunicación y enseñanza de las técnicas, así como una expansión y desarrollo de las mismas.

Actualmente, se plantea que en determinados procedimientos quirúrgicos podría llegar a ser un factor independiente de mejor evolución de los pacientes[1]. En Uruguay, así como en Latinoamérica, la incorporación de la ecografía si bien fue precoz, ha estado limitada por diferentes motivos, como es el lento acceso a la tecnología necesaria para la adquisición de las habilidades requeridas y, por lo tanto, el incompleto o nulo entrenamiento de los anestesiólogos[2].

Un reciente artículo[3] puso en evidencia nuestra realidad en dos aspectos fundamentales: 1) la baja utilización de bloqueos nerviosos periféricos y, 2) que la técnica de neurolocalización más utilizada sigue siendo la neuroestimulación.

Según este relevamiento realizado en Latinoamérica, los escasos programas de residencia que incluían la anestesia regional en su currícula (ecoguiada, aún incipiente y con neuroestimulación, la gran mayoría), representaban en ese momento el $45 \%$ de las posibilidades formativas disponibles. Terminada la etapa formativa, egresados ya de las tutorías curriculares, las oportunidades académicas eran, en la mejor perspectiva, escasas, incompletas y difíciles de realizar cabalmente.

Frente a esta realidad y siendo la Facultad de Medicina de la Universidad de la República, el único formador de recursos humanos en anestesiología, se incorporó a la currícula del residente, un curso específico de anestesia regional con integración de habilidades en bloqueos regionales periféricos ecoguiados.

En Uruguay, durante la residencia de Anestesiología se deben aprobar 8 semestres en un período de 4 años. Este curso fue incorporado en el segundo año de formación de la residencia, en el contexto de la enseñanza de la Anestesia Regional. Comprende las bases teóricas, que las integra al entrenamiento de las ineludibles habilidades prácticas necesarias, con control de docentes tutores y evaluación curricular.

Cumplido el año de instaurado este programa formativo, nos propusimos evaluar las actividades comprendidas en el período entre abril de 2017 y marzo de 2018.

\footnotetext{
Asistente de Anestesiología.

Profesor Agregado.

Docente Asociado.

Departamento de Anestesiología. Hospital de Clínicas. Facultad de Medicina

Universidad de la República del Uruguay.
}

Fecha de recepción: 14 de septiembre de 2018

Fecha de aceptación: 22 de septiembre de 2018

\section{ORCID}

https://orcid.org/0000-0002-0761-3251

\section{Correspondencia:}

Profesor Agregado. Juan Bouchacourt

Email: jboucha@hc.edu.uy 
Se realizó la rotación teórico-práctica en 4 centros con alto número de práctica de técnicas regionales y con la de incorporación como docentes asociados a especialistas anestesiólogos con experiencia docente en la realización de estas técnicas regionales.

En este período cumplieron con el curso 13 residentes. La carga horaria para habilidades y destrezas fueron 12 horas semanales para el desarrollo de la técnica regional con supervisión en quirófano. La evaluación de los aspectos prácticos se realizó a través de una planilla que detalla los bloqueos realizados y el resultado de los mismos. En la Tabla 1 se muestran los resultados brutos de la experiencia.

Se completó la evaluación de 12 residentes, que realizaron un total de 780 bloqueos. La media de bloqueos fue de $65 \pm 60$ con un máximo individual de 226 y un mínimo de 14.

Discriminados por topografía, existió un claro predominio de bloqueos de miembros inferiores con un $91,7 \%$, en comparación con los de miembro superior que fueron un $7,5 \%$, y un $0,65 \%$ correspondieron a bloqueos de pared abdominal.

Dentro de los bloqueos de miembro inferior, los más realizados fueron el del nervio femoral $(38,1 \%)$, el de las ramas del nervio obturador $(26,1 \%$ ) y el nervio femorocutáneo (18,6\%).

En cuanto a los bloqueos de miembro superior, los más realizados fueron el abordaje infraclavicular del plexo braquial (35\%), el abordaje supraclavicular del plexo braquial (22\%), el nervio mediano $(6,7 \%)$, el plexo braquial a nivel axilar y el nervio cubital (5\%).

No se registraron complicaciones graves derivadas de las técnicas realizadas durante la rotación tutelada.

\section{Conclusiones de los resultados preliminares}

Esta evaluación nos mostró:

- En primer lugar, la realidad de expansión de las oportunidades educativas en centros no vinculados administrativamente a la Universidad de la República, bajo la rectoría docente del Departamento de Anestesiología, mediante docentes específicamente asociados para este fin, lo que permitió llevar adelante el curso.

- Que, aún en la multiplicidad de los centros de ejecución del curso, la planificación académica, única, curricular de los objetivos, temario, evaluación y documentación, nos permitió cuantificar la adquisición de habilidades y destrezas para este tipo de procedimientos.

- Consecuentemente pudimos establecer cantidad y tipos de bloqueos que pudimos entrenar, establecer nuestros planes de desarrollo y nuevos objetivos.

- De acuerdo a estudios realizados dentro y fuera de nuestra región, nuestros resultados en cuanto a lo numérico son coincidentes. No obstante, a diferencia de estos, encontramos un franco predominio de los bloqueos de miembros inferiores

Tabla 1. Bloqueos realizados en el período desde abril de 2017 a marzo de 2018

\begin{tabular}{lcc}
\hline Tipo de bloqueo & $\mathbf{n}$ & $\%$ \\
Femoral & 273 & 35,0 \\
Femorocutáneo & 133 & 17,1 \\
Obturador & 187 & 24,0 \\
Ciático & 90 & 11,5 \\
Canal aductores & 25 & 3,2 \\
Cuello de pie & 5 & 0,6 \\
Otros bloqueos miembro inferior & 3 & 0,4 \\
Interescalénico & 15 & 1,9 \\
Supraclavicular & 13 & 1,7 \\
Infraclavicular & 19 & 2,4 \\
Axilar & 3 & 0,4 \\
Bloqueos distales miembro superior & 8 & 1,0 \\
TAP Block & 5 & 0,6 \\
Total 780 bloqueos & & . \\
\hline
\end{tabular}


vinculado a las tendencias quirúrgicas de los centros de entrenamiento[4],[5].

- En tercer lugar nos permitió tener un punto de partida para convalidar algunos aspectos y corregir otros, dentro de la dinámica vertiginosa que hoy impone al sistema educativo, las necesidades del sistema de salud y las nuevas tecnologías.

En suma, en Uruguay, la incorporación curricular de formación y entrenamiento en técnicas regionales, unificada académicamente desde el Departamento de Anestesiología, nos permitió un cambio sustantivo en los recursos docentes y materiales específicos, con incorporación real, evaluada, de conocimientos y habilidades técnicas de los especialistas en formación.

También, la documentación de esta actividad, nos permitió cuantificar la realización de la misma y establecer una amplia estrategia para responder a las necesidades de la especialidad en esta materia.

Una de las limitaciones a corregir en el futuro es lograr establecer el número de casos mínimo necesario para un éxito adecuado en términos de eficiencia y así definir las curvas de aprendizajes institucionales e individuales lo que constituye una herramienta relevante.

\section{Referencias}

1. Guay J, Parker MJ, Griffiths R, Kopp SL. Peripheral Nerve Blocks for Hip Fractures: A Cochrane Review. Anesth Analg. 2018 May;126(5):1695704. https://doi.org/10.1213/ ANE.0000000000002489 PMID:28991122

2. Altermatt FR, Corvetto MA, Mc Cready $\mathrm{M}$, Altermatt F. Práctica de la anestesia regional en Chile.
Encuesta on line. Rev Chil Anest. 2007;36:246.

3. Corvetto MA, Carmona J, Vásquez MI, Salgueiro C, Crostón J, Sosa R, et al. Práctica actual de la anestesia regional en América del Sur: encuesta en línea. Rev Esp Anestesiol Reanim. 2017 Jan;64(1):27-31. https://doi.org/10.1016/j. redar.2016.05.001 PMID:27377713

4. Schupfer G. Monitoring the adquisition of competences in anesthesia. Leuven: Update in Anesthesia; 2006.

5. Konrad C, Schüpfer G, Wietlisbach M, Gerber H. Learning manual skills in anesthesiology: is there a recommended number of cases for anesthetic procedures? Anesth Analg. 1998 Mar;86(3):635-9. https:// doi.org/10.1213/00000539199803000-00037 PMID:9495429 